\title{
CONGRUENCES FOR COEFFICIENTS OF DRINFELD MODULAR FORMS
}

\author{
SoYoung CHOI
}

\begin{abstract}
We find congruences for the $t$-expansion coefficients of a Drinfeld modular form for $\Gamma_{0}^{+}(Q)$, where $Q$ is a monic irreducible polynomial. As an application we obtain new congruence relations for the $t$-expansion coefficients of Drinfeld modular forms for $G L_{2}\left(\mathbb{F}_{q}[T]\right)$.
\end{abstract}

\section{Introduction}

By using the action of the Hecke operators Gekeler [4] and López [7] proved the existence of congruences for the coefficients of two distinguished Drinfeld modular forms, the Poincaré series $P_{q+1,1}$ and the discriminant function $\Delta$, respectively. Gallardo and López [2] showed that there exist congruences for the $s$-expansion coefficients of the Eisenstein series of weight $q^{k}-1$ for any positive integer $k$. By using the residue theorem, we [1] found divisibility properties for $t$-expansion coefficients of Drinfeld modular forms for $G L_{2}\left(\mathbb{F}_{q}[T]\right)$ and as a consequence we obtained further congruence relations.

Let $Q$ be a monic irreducible polynomial in $\mathbb{F}_{q}[T]$. Let $\Gamma_{0}(Q)$ be the subgroup of $G L_{2}\left(\mathbb{F}_{q}[T]\right)$ of matrices that are congruent to $\left(\begin{array}{c}* * \\ 0 *\end{array}\right)$ modulo $Q$. Let $\Gamma_{0}^{+}(Q)$ be the subgroup of $G L_{2}\left(\mathbb{F}_{q}(T)\right)$ generated by $\Gamma_{0}(Q)$ and the Atkin-Lehner involution $W_{Q}:=\left(\begin{array}{cc}0 & -1 \\ Q & 0\end{array}\right)$. Let $X_{\Gamma_{0}^{+}(Q)}$ be the compactification of $\Gamma_{0}^{+}(Q) \backslash \Omega$, where $\Omega$ is the Drinfeld upper half-plane.

In this paper we define Drinfeld modular forms for $\Gamma_{0}^{+}(Q)$ of weight $k$ and type $m$. Let $M_{k}^{m}\left(\Gamma_{0}^{+}(Q)\right)$ be the $C$-vector space of such Drinfeld modular forms. Then $M_{k}^{m}\left(\Gamma_{0}^{+}(Q)\right)$ is a subspace of the space of Drinfeld modular forms for $\Gamma_{0}(Q)$ of weight $k$ and type $m$. We generalize results in [1] to these subspaces.

By using the residue theorem on the curve $X_{\Gamma_{0}^{+}(Q)}$ we find congruences for the $t$-expansion coefficients of Drinfeld modular forms for $\Gamma_{0}^{+}(Q)$ when $q$ is odd (Theorem 3.3). As an application, we obtain new congruence relations for the coefficients of Drinfeld modular forms for the modular group $G L_{2}\left(\mathbb{F}_{q}[T]\right)$ (Theorem 3.6).

Received April 3, 2008.

2000 Mathematics Subject Classification. 11F52

Key words and phrases. Drinfeld modular form, congruence. 


\section{Preliminary}

Let $A$ be the ring $\mathbb{F}_{q}[T]$ of polynomials over a finite field $\mathbb{F}_{q}$ and $K$ the rational function field $\mathbb{F}_{q}(T)$ over $\mathbb{F}_{q}$. Let $C$ be the completion of the algebraic closure of the completion, $K_{\infty}$, of $K$ at $1 / T$ and $\Omega:=C-K_{\infty}$ the Drinfeld upper half plane. Then the group $G L_{2}(K)$ acts on $\Omega$ in the following way: if $\gamma=\left(\begin{array}{ll}a & b \\ c & d\end{array}\right) \in G L_{2}(K)$ and $z \in \Omega$, then

$$
\gamma z=\frac{a z+b}{c z+d}
$$

Let $L=\tilde{\pi} A$ be the rank $1 A$-lattice in $C$ associated to the Carlitz module $\rho$,

$$
\rho_{T}(X)=T X+X^{q} .
$$

Let $e_{L}$ be the exponential function associated to $L$, i.e.,

$$
e_{L}: C \rightarrow C, \quad e_{L}(z)=z \prod_{\lambda \in L-\{0\}}\left(1-\frac{z}{\lambda}\right)
$$

We let $t=t(z):=1 /\left(\tilde{\pi} e_{A}(z)\right)$ and $s=s(z):=t(z)^{q-1}$. For any nonzero $a \in A$ we define $t_{a}=t_{a}(z):=t(a z)$ and the $a$-th inverse cyclotomic polynomial $f_{a}(X) \in A[X]$ by

Then we have $t_{a}=t^{q^{\operatorname{deg} a}} / f_{a}(t)$.

$$
f_{a}(X)=\rho_{a}\left(X^{-1}\right) X^{q^{\operatorname{deg} a}}
$$

Hereafter $Q$ is a monic irreducible polynomial in $A$. Let $\Gamma_{0}^{+}(Q)$ be the subgroup of $G L_{2}(K)$ generated by $\Gamma_{0}(Q)$ and the Atkin-Lehner involution $W_{Q}:=\left(\begin{array}{cc}0 & -1 \\ Q & 0\end{array}\right)$. Let $X_{\Gamma_{0}^{+}(Q)}$ be the compactification of $\Gamma_{0}^{+}(Q) \backslash \Omega$ and $X_{\Gamma_{0}^{+}(Q)}-$ $\Gamma_{0}^{+}(Q) \backslash \Omega:=\{\infty\}$.

For a 1-form $\omega$ on $X_{\Gamma_{0}^{+}(Q)}$ we have the residue theorem ([6, Theorem 7.14.2]) as follows

$$
\sum_{p \in X_{\Gamma_{0}^{+}(Q)}} \operatorname{Res}_{p} \omega=0 .
$$

Lemma 2.1. $s$ is a uniformizing parameter at $\infty$ for $X_{\Gamma_{0}^{+}(Q)}$.

Proof. This is the same proof as (2.7) in [5].

\section{Congruences for coefficients of Drinfeld modular forms for $\Gamma_{0}^{+}(Q)$}

We can define a Drinfeld modular form for $\Gamma_{0}^{+}(Q)$ of weight $k$ and type $m$ as follows: The usual slash operator $\left.f\right|_{k} ^{m} \gamma$ is defined as

$$
\left(\left.f\right|_{k} ^{m} \gamma\right)(z):=(\operatorname{det} \gamma)^{m}(c z+d)^{-k} f(\gamma z)
$$

where $\gamma=\left(\begin{array}{ll}a & b \\ c & d\end{array}\right) \in G L_{2}(K)$.

A (respectively meromorphic) Drinfeld modular form for $\Gamma_{0}^{+}(Q)$ of weight $k$ and type $m$ (where $k \geq 0$ and $m$ are integers) is a holomorphic (respectively meromorphic) function $f: \Omega \rightarrow C$ that satisfies: 
(i) $\left(\left.f\right|_{k} ^{m} \gamma\right)(z)=f(z)$ for any $\gamma=\left(\begin{array}{ll}a & b \\ c & d\end{array}\right) \in \Gamma_{0}^{+}(Q)$,

(ii) $f$ is holomorphic (respectively meromorphic) at the cusp $\infty$.

The condition (ii) says that $f$ has a power series (respectively Laurent series) expansion at the cusp. If $f$ is a meromorphic Drinfeld modular form for $\Gamma_{0}^{+}(Q)$ of weight $k$ and type $m$, then the $t$-expansion of $f$ is of the form

$$
f=\sum_{i} a_{f}((q-1) i+m) t^{(q-1) i+m}
$$

Moreover, it becomes a meromorphic Drinfeld modular form for $\Gamma_{0}(Q)$ of weight $k$ and type $m$. Let $M_{k}^{m}\left(\Gamma_{0}^{+}(Q)\right)$ be the $C$-vector space of Drinfeld modular forms for $\Gamma_{0}^{+}(Q)$ of weight $k$ and type $m$. We assume that $k=2 m$ is an even nonnegative integer. If not, the space $M_{k}^{m}\left(\Gamma_{0}^{+}(Q)\right)$ is the zero space. Indeed, since $\left(\begin{array}{cc}-Q & 0 \\ 0 & -Q\end{array}\right) \in \Gamma_{0}^{+}(Q), f$ is invariant under the action of $\left(\begin{array}{cc}-Q & 0 \\ 0 & -Q\end{array}\right)$ by the slash operator, that is, $f(z)=Q^{-2 m}(-Q)^{k} f(z)$, which implies that $k=2 m$ if $f(z) \neq 0$.

Example 3.1. If $k=2 m$ and $f$ is a meromorphic Drinfeld modular form for $G L_{2}(A)$ of weight $k$ and type $m$, then $f(z)+Q^{m} f(Q z)$ is a meromorphic Drinfeld modular form for $\Gamma_{0}^{+}(Q)$ of weight $k$ and type $m$.

For any meromorphic Drinfeld modular form $G(z)$ for $\Gamma_{0}^{+}(Q)$ of weight 2 and type $1, \omega:=G(z) d z$ can be considered as a 1 -from on $X_{\Gamma_{0}^{+}(Q)}$. Let

$$
G(z)=\sum_{i=n_{0}}^{\infty} a((q-1) i+1) t^{(q-1) i+1}
$$

be the $t$-expansion of $G(z)$ at $\infty$ and

$$
\pi: \Omega \rightarrow \Gamma_{0}^{+}(Q) \backslash \Omega
$$

be the quotient map. For any point $\tau \in \Omega$, let

$$
e_{\tau}=\left|\Gamma_{0}^{+}(Q)_{\tau} /\left(\Gamma_{0}^{+}(Q)_{\tau} \cap Z(K)\right)\right|,
$$

where $\Gamma_{0}^{+}(Q)_{\tau}$ is the stabilizer of $\tau$ in $\Gamma_{0}^{+}(Q)$ and $Z(K)$ is the center of scalar matrices.

In what follows we suppose that $q=p^{l}$ is odd. Then $e_{\tau}$ is prime to $p$ because $e_{\tau}$ equals $n_{\tau}$ or $2 n_{\tau}$, where $n_{\tau}=\left|\Gamma_{0}(Q)_{\tau} /\left(\Gamma_{0}(Q)_{\tau} \cap Z(K)\right)\right|$ has the value 1 or $q+1$. Moreover we have the following.

\section{Lemma 3.2 .}

(i) $\operatorname{Res}_{\infty} \omega=-a(1) / \widetilde{\pi}$.

(ii) $\operatorname{Res}_{\pi(\tau)} \omega=e_{\tau}^{-1} \operatorname{Res}_{\tau} G(z)$ for each $\tau \in \Omega$.

Proof. (i) follows from the simple fact that $d t=-\tilde{\pi} t^{2} d z$. Let $\tau \in \Omega$. We choose uniformizers $x$ and $y$ on $\Omega$ and $\Gamma_{0}^{+}(Q) \backslash \Omega$, respectively, with $y=x^{e_{\tau}}$. Then $d y=e_{\tau} x^{e_{\tau}-1} d x$, which gives the assertion (ii). 
To state the main theorem we let

$$
\rho_{Q}(t)=\sum_{0 \leq j \leq \operatorname{deg} Q} l_{j} t^{q^{j}} .
$$

Then for a nonnegative integer $a$,

$$
1 / t(Q z)^{p^{a}}=\left(\frac{f_{Q}(t)}{t^{q^{\operatorname{deg} Q}}}\right)^{p^{a}}=\rho_{Q}\left(t^{-1}\right)^{p^{a}}=\sum_{0 \leq j \leq \operatorname{deg} Q} l_{j}^{p^{a}} t^{-p^{a} q^{j}} .
$$

Theorem 3.3. Suppose that $k=2 m$ and $q$ is odd. Let $\bar{m}$ be the nonnegative integer such that $m \equiv \bar{m} \bmod (q-1)$ and $0 \leq \bar{m}<q-1$ and let

$$
r:=\operatorname{dim}_{C} M_{k}^{m}\left(\Gamma_{0}(1)\right)=\left[\frac{k-(q+1) \bar{m}}{q^{2}-1}\right]+1 \quad(\text { see }[1]) .
$$

Let $f \in M_{k}^{m}\left(\Gamma_{0}^{+}(Q)\right)$ have the $t$-expansion as follows

$$
f=\sum_{i \geq-m /(q-1)} a_{f}((q-1) i+m) t^{(q-1) i+m} \in O_{L}[[t]],
$$

where $L$ is a finite algebraic extension field of $K$ with ring $O_{L}$ of integers.

For any integer a such that $p^{a}+1 \geq \bar{m}+r(q-1)$ and $\bar{m} \equiv p^{a}+1 \bmod$ $(q-1)$, we have that

$$
\begin{aligned}
& Q^{m-1} a_{f}\left((q-1) \frac{p^{a}+1-m}{q-1}+m\right) \\
& +\sum_{0 \leq j \leq \operatorname{deg} Q} l_{j}^{p^{a}} a_{f}\left((q-1) \frac{p^{a} q^{j}+1-m}{q-1}+m\right) \equiv 0 \bmod \left(T^{q}-T\right) .
\end{aligned}
$$

Proof. Let

$$
\alpha:=\frac{k-2 \bar{m}}{q-1}+(1-r)(q+1)-\bar{m} \quad \text { and } \quad l_{a}:=\frac{p^{a}+1-\bar{m}}{q-1}-r .
$$

Let $h$ be the Pincaré series $P_{q+1,1}$ and $g$ the normalized Eisenstein series of weight $q-1$. Noticing that we can consider $h^{(q-1)\left(r+l_{a}\right)+\bar{m}-1}$ as a Drinfeld modular form for $G L_{2}(A)$ of type $m-1$ and $g^{(q+1) l_{a}-\alpha+q}$ as a Drinfeld modular form for $G L_{2}(A)$ of type 0 , we choose

$$
B(z):=\frac{g^{(q+1) l_{a}-\alpha+q}}{h^{(q-1)\left(r+l_{a}\right)+\bar{m}-1}} \quad \text { and } \quad C(z):=B(Q z),
$$

which are of weight $2-k$ and

$$
F(z):=Q^{m-1} B(z) f(z)+C(z) f(z) .
$$


Then clearly we have $\left(\left.\left(Q^{m-1} B+C\right)\right|_{2-k} ^{1-m} \gamma\right)(z)=Q^{m-1} B(z)+B(Q z)$ for any $\gamma \in \Gamma_{0}(Q)$. Moreover,

$$
\begin{aligned}
& \left(\left.\left(Q^{m-1} B+C\right)\right|_{2-k} ^{1-m}\left(\begin{array}{cc}
0 & -1 \\
Q & 0
\end{array}\right)\right)(z) \\
= & \left(\left.Q^{m-1} B\right|_{2-k} ^{1-m}\left(\begin{array}{cc}
0 & -1 \\
Q & 0
\end{array}\right)\right)(z)+\left(\left.C\right|_{2-k} ^{1-m}\left(\begin{array}{cc}
0 & -1 \\
Q & 0
\end{array}\right)\right)(z) \\
= & Q^{1-m} Q^{m-1}(Q z)^{k-2} B\left(\frac{-1}{Q z}\right)+Q^{1-m}(Q z)^{k-2} B\left(\frac{-1}{z}\right) \\
= & B(Q z)+Q^{m-1} B(z) .
\end{aligned}
$$

Hence $B(Q z)+Q^{m-1} B(z)$ is invariant under the action of $\Gamma_{0}^{+}(Q)$ by the slash operator, which implies that $F(z)$ is a meromorphic Drinfeld modular form of weight 2 and type 1 for $\Gamma_{0}^{+}(Q)$. Since $F(z)$ is holomorphic on $\Omega$, the residue theorem on $X_{\Gamma_{0}^{+}(Q)}$ implies that

$$
\sum_{p \in X_{\Gamma_{0}^{+}(Q)}} \operatorname{Res}_{p} F(z) d z=\operatorname{Res}_{\infty} F(z) d z=0 .
$$

Moreover note that $g \equiv 1 \bmod \left(T^{q}-T\right)$ and $h=-t-t^{(q-1)^{2}-1}+\cdots$ Cor. 10.4]). Therefore, the coefficient of $t$ in

$$
\begin{aligned}
& Q^{m-1} \frac{g^{(q+1) l_{a}-\alpha+q} f}{h^{(q-1)\left(r+l_{a}\right)+\bar{m}-1}}+\frac{g(Q z)^{(q+1) l_{a}-\alpha+q} f}{h(Q z)^{(q-1)\left(r+l_{a}\right)+\bar{m}-1}} \quad\left(\in M_{2}^{1}\left(\Gamma_{0}^{+}(Q)\right)\right) \\
\equiv & Q^{m-1} \frac{f}{h^{p^{a}}}+\frac{f}{h(Q z)^{p^{a}}} \bmod \left(T^{q}-T\right) \\
\equiv & Q^{m-1}\left(\sum_{i \geq-m /(q-1)} a_{f}((q-1) i+m) t^{(q-1) i+m}\right)\left(\frac{-1}{t^{p^{a}}}+t^{\left((q-1)^{2}-1\right) p^{a}}+\cdots\right) \\
+ & \left(\sum_{i \geq-m /(q-1)} a_{f}((q-1) i+m) t^{((q-1) i+m}\right)\left(\frac{-1}{t(Q z)^{p^{a}}}+t(Q z)^{\left.(q-1)^{2}-1\right) p^{a}}+\cdots\right) \\
& \bmod \left(T^{q}-T\right)
\end{aligned}
$$

is zero. This gives the assertions.

Remark 3.4. Note that for $f, m$ is necessarily larger than 1 .

We obtain the following result.

Corollary 3.5. With the notations and assumptions of Theorem 3.3 we have that for any monic irreducible polynomial $Q$ of degree 1 in $A$,

$$
a_{f}\left((q-1) \frac{p^{a} q+1-m}{q-1}+m\right) \equiv 0 \bmod Q .
$$

Now by using theorem 3.3 we obtain new congruence relations for the $t$ expansion coefficients of Drinfeld modular forms for $G L_{2}\left(\mathbb{F}_{q}[T]\right)$ as follows. 
Theorem 3.6. Let $k, m, q, \bar{m}$ and $r$ be as in Theorem 3.3 and suppose that $f \in M_{k}^{m}\left(\Gamma_{0}(1)\right)$ has the $t$-expansion as follows

$$
f=\sum_{i \geq-m /(q-1)} a_{f}((q-1) i+m) t^{(q-1) i+m} \in O_{L}[[t]],
$$

where $L$ is a finite algebraic extension field of $K$ with ring $O_{L}$ of integers.

For any integer a such that $p^{a}+1 \geq \bar{m}+r(q-1)$ and $\bar{m} \equiv p^{a}+1 \bmod$ $(q-1)$, we have that for any monic irreducible polynomial $Q$ of degree 1 in $A$,

$$
a_{f}\left((q-1) \frac{p^{a} q+1-m}{q-1}+m\right) \equiv 0 \bmod Q .
$$

Proof. For $f \in M_{k}^{m}\left(\Gamma_{0}(1)\right)$, we obtain that $f(z)+Q^{m} f(Q z) \in M_{k}^{m}\left(\Gamma_{0}^{+}(Q)\right)$. Since $f(z)+Q^{m} f(Q z) \equiv f(z) \bmod Q$, by Theorem 3.3 and Remark 3.4, we have that

$$
\begin{aligned}
& \sum_{0 \leq j \leq 1} l_{j}^{p^{a}} a_{f}\left((q-1) \frac{p^{a} q^{j}+1-m}{q-1}+m\right) \\
\equiv & a_{f}\left((q-1) \frac{p^{a} q+1-m}{q-1}+m\right) \equiv 0 \bmod Q .
\end{aligned}
$$

Acknowledgment. The author thanks a referee for revising this paper.

\section{References}

[1] S. Choi, Linear relations and congruences for the coefficients of Drinfeld modular forms, Israel J. Math. 165 (2008), 93-101.

[2] J. Gallardo and B. López, "Weak" congruences for coefficients of the Eisenstein series for $\mathbb{F}_{q}[T]$ of weight $q^{k}-1$, J. Number Theory 102 (2003), no. 1, 107-117.

[3] E. U. Gekeler, On the coefficients of Drinfel?d modular forms, Invent. Math. 93 (1988), no. 3, 667-700.

[4] - Growth order and congruences of coefficients of the Drinfeld discriminant function, J. Number Theory 77 (1999), no. 2, 314-325.

[5] E. U. Gekeler and M. Reversat, Jacobians of Drinfeld modular curves, J. Reine Angew. Math. 476 (1996), 27-93.

[6] R. Hartshorne, Algebraic Geometry, Graduate Texts in Mathematics, No. 52. SpringerVerlag, New York-Heidelberg, 1977.

[7] B. López, A congruence for the coefficients of the Drinfeld discriminant function, C. R. Acad. Sci. Paris Ser. I Math. 330 (2000), no. 12, 1053-1058.

Department of Mathematics Education

DONGGUK UNIVERSITY

Gyeonguu 780-714, Korea

E-mail address: young@dongguk.ac.kr 